\title{
New incoherent scatter diagnostic methods for the heated D-region ionosphere
}

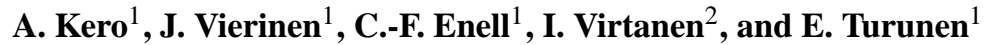 \\ ${ }^{1}$ Sodankylä Geophysical Observatory, University of Oulu, Sodankylä, Finland \\ ${ }^{2}$ Dept. of Physical Sciences, University of Oulu, Finland
}

Received: 4 January 2008 - Revised: 17 April 2008 - Accepted: 19 May 2008 - Published: 5 August 2008

\begin{abstract}
A new incoherent scatter (IS) diagnostic technique for the actively heated D-region ionosphere is presented. In this approach, an exponential autocorrelation function (ACF) was fitted to the data by using a Markov Chain Monte Carlo (MCMC) inversion and the Sodankylä Ion Chemistry model (SIC). The method was applied for a set of combined EISCAT heating and VHF radar experiments carried out in November 2006. A newly designed radar experiment, sippi, based on optimised phase codes and direct sampling of the transmitted and the received IS signal, was used to produce ACF estimates of the target plasma. Systematic features associated with the heating were found by comparing the mean ACFs corresponding to heated and unheated periods. However, the data analysis revealed reasonable electron temperature estimates at the altitudes of the expected maximum effect (70-75 km) only in three cases out of six, corresponding to the highest signal-to-noise ratio (SNR) conditions. In two of these cases, the electron temperature was increased by a factor of 5 to 7 , which is in a good agreement with the theoretical heating modelling. In the case of the first presented data example, the model overestimates the effect. These are the first successful IS observations of the HF-induced maximum electron temperature enhancements in the D-region ionosphere.
\end{abstract}

Keywords. Ionosphere (Active experiments; Ion chemistry and composition; Instruments and techniques)

\section{Introduction}

Powerful radio waves can heat an electron gas via collisions between the electrons and neutral particles. This effect is known to take place in the D-region ionosphere since discovering the Luxembourg effect (Bailey and Martyn, 1934). Ac-

Correspondence to: A. Kero

(antti.kero@sgo.fi) cording to theoretical models (Belova et al., 1995; Kero et al., 2000), the EISCAT heater facility (Rietveld et al., 1992) is capable of increasing the electron temperature by a factor of 5-10 in the D region, depending on ionospheric conditions. Various evidence for the existence of the heater induced Dregion effect is available including successful modification of ionospheric conductivities (e.g. Stubbe et al., 1982; Barr et al., 1984; Rietveld et al., 1986) and mesospheric chemistry (e.g. Rietveld et al., 1996; Chilson et al., 2000). However, an experimental quantification of the electron temperature at its maximum in the heated D-region ionosphere is still missing. In particular, incoherent scatter (IS) radars should be able to observe directly the plasma parameters, such as electron temperature. However, the heated D-region ionosphere is not a trivial target for the IS radar technique because of the low signal-to-noise ratio (SNR) due to low electron density and the Lorentzian shape of the IS spectrum due to high electron to neutral collision frequency. In fact, there is only one study so far showing estimates for heated D-region electron temperatures based on IS measurements (Kero et al., 2000). However, according to theoretical modelling, the maximum heating effect appeared below the altitudes of sufficient SNR in this case.

In November 2006, three daytime experiments were carried out in order to detect the D-region heating effect by using the EISCAT VHF radar and the heater. The so-called sippi experiment, using optimised phase codes and direct sampling of the transmitted and the received signal, probed the D-region ionosphere which was heated in 6-s on/off intervals. An ambiguity free autocorrelation function (ACF) estimate of the target plasma was calculated for both heated and unheated periods based on the recorded samples (Virtanen et al., 2008).

A new IS data-analysis method, based on Markov Chain Monte Carlo (MCMC) inversion of the IS theory for a collisional plasma, was applied to the measurements. The MCMC method has several advantages compared to other

Published by Copernicus Publications on behalf of the European Geosciences Union. 

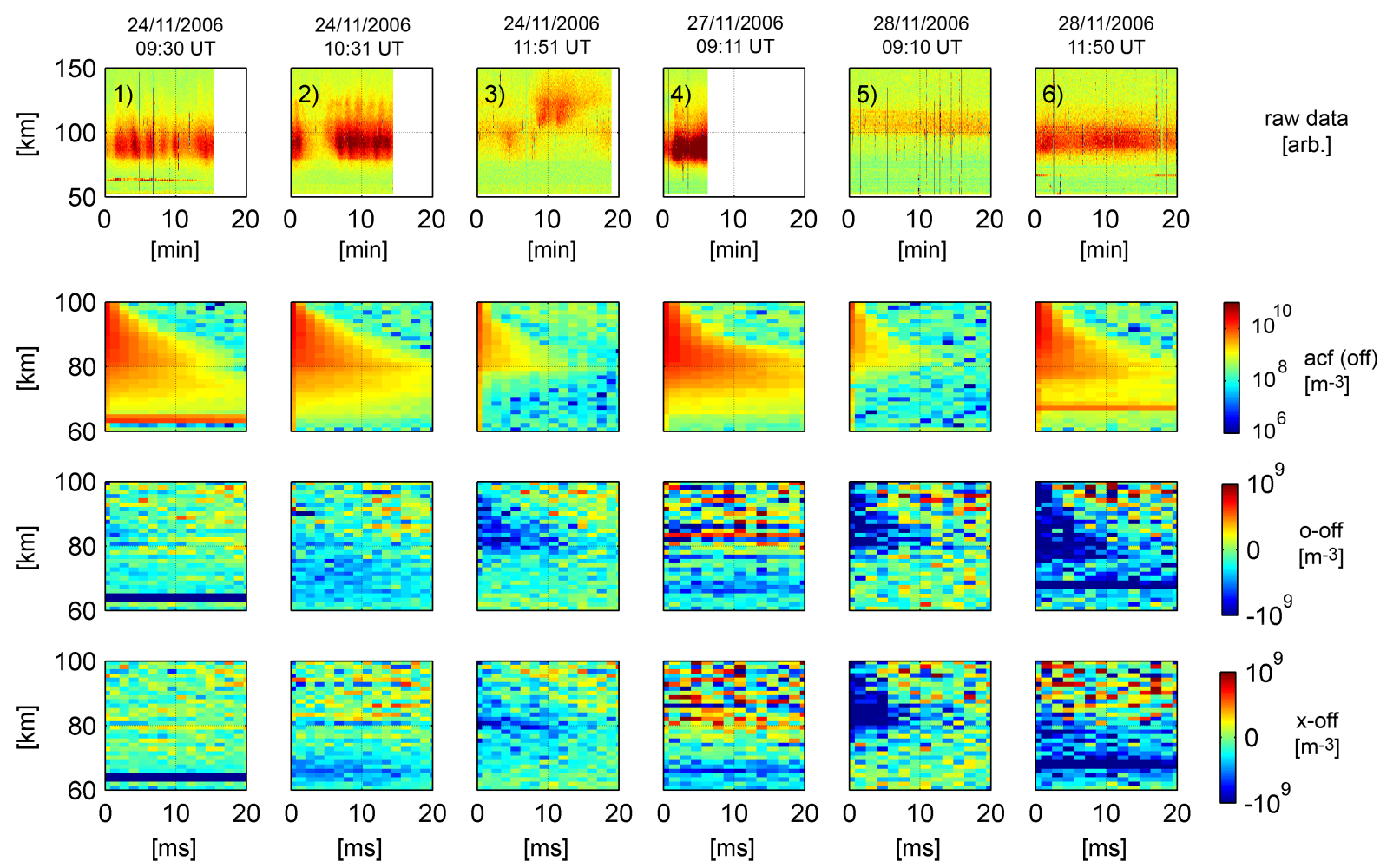

Fig. 1. Measured data in the 6 cases studied. First row of panels: raw un-calibrated IS power (50-150 km) as a function of time. Each window is $20 \mathrm{~min}$ long. Second row: mean ACF (logarithmic scaled absolute value) corresponding to heater off-periods. Zero-lag power is calibrated to the electron density at $120 \mathrm{~km}$ by using Troms $\varnothing$ Dynasonde. Third row: mean ACF difference between heater o-mode and heat-off periods. Last row: mean ACF difference between x-mode and off periods.

methods for exploring the probability distribution. Instead of searching for the most probable model parameters, it estimates the full distribution of the parameters by directly sampling the distribution providing the real distribution of parameter estimation errors. The method is also less vulnerable to local maxima of the probability distribution than methods that only seek the maximum of the probability distribution.

The theoretical response of the ACF to the heating, via electron-temperature-dependent Debye length and ion chemistry, was parameterised by using the Sodankylä Ion Chemistry (SIC) model (Turunen et al., 1996).

\section{Data}

Three combined EISCAT heating and daytime VHF radar experiments were carried out in November 2006 in order to detect the D-region heating effect. The heater parameters used were selected to maximise the radio wave absorption and make it possible to compare the two polarisations (oand $\mathrm{x}$-mode). Antenna array 1 was used with full power at $5.423 \mathrm{MHz}$ frequency providing an effective radiative power of $600 \mathrm{MW}$. The heater modulation used was o-mode/off/xmode/off in 6-s intervals.
For the VHF radar, the newly designed sippi experiment was used. Instead of a traditional hardware correlator based ACF measurement, transmitted and received signals were sampled directly with high-speed AD converters and stored on hard disks. As a result, an ambiguity-free ACF was determined by applying statistical inversion to the recorded signals (Virtanen et al., 2008). One of the major advantages of this approach, particulary in the application of high power heating, is that all unwanted interference between the heater and the radar via power lines are canceled out automatically in the analysis.

Every more than 5 min long continuous period of sippi data, using the heater modulation described above, are shown in Fig. 1. These six periods were analysed with 2-s time resolution synchronised to the heating cycle. The IS power profiles (zero-lag estimates of ACF) were decoded up to $150 \mathrm{~km}$, while the time-demanding full calculation of ACF was done only up to $100 \mathrm{~km}$ altitude. After manual removal of clearly bad individual ACF profiles, such as contaminated by meteors, the data was averaged separately over the o-, $\mathrm{x}$ - and off-mode periods. These ACF profiles were scaled into $\mathrm{m}^{-3}$ units by 1) removing the spatial $1 / r^{2}$-factor and 2) calibrating the IS power to the Tromsø Dynasonde electron density at $120 \mathrm{~km}$ altitude (see Fig. 2). 

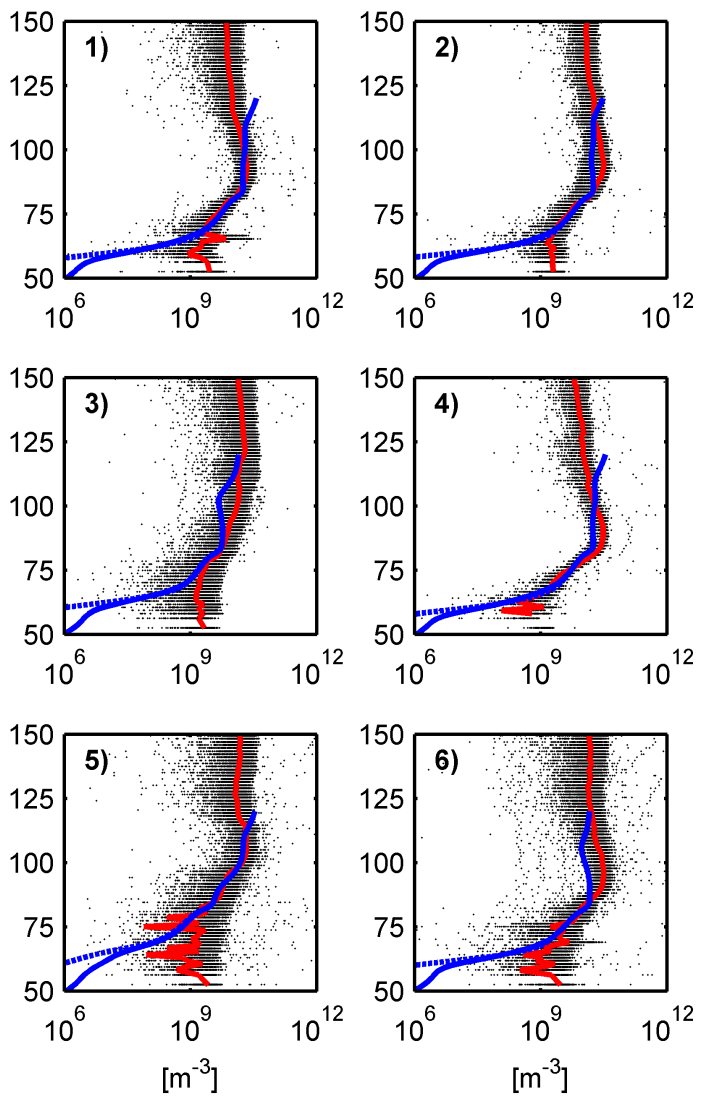

Fig. 2. Detected IS power vs the SIC model for the six cases studied. Red line: mean value of the IS power estimates (black dots) corresponding to heater off-periods. Blue lines: SIC modelled electron density (continuous) and theoretical IS power (Eq. 2) based on the SIC modelled $N_{e}, \lambda$ and $m_{i}$ (dashed).

Figure 1 shows a systematic difference in the mean ACF between the heater on and off periods: the strength of the ACF turns out to be weaker in the heated ionosphere. Moreover, this signature appears at different heights depending on ionospheric conditions. Figure 2 shows that the studied data examples can be divided roughly in two groups according to the SNR: cases 1, 2 and 4 show less variance in the IS power compared to the other cases which represent somewhat lesser electron densities at $75 \mathrm{~km}$ altitude. The heating signature in Fig. 1 appears at lower altitudes in the high electron density conditions compared to the weaker D-region cases 3 and 5. Note also, that the $\mathrm{x}$-mode seems to produce somewhat narrower effect compared to the o-mode signature. The behaviour follows roughly the modelled heating effect altitude profiles (see Figs. 4 and 5).

In addition, there is a clearly visible Polar Mesospheric Winter Echo (PMWE) in the cases 1 and 6, and a hint of PMWE is present also in the cases 2 and 4 (Fig. 1, the second row of panels, $65-70 \mathrm{~km}$ ). The average IS power at the PMWE layer during the heater on period is roughly half of

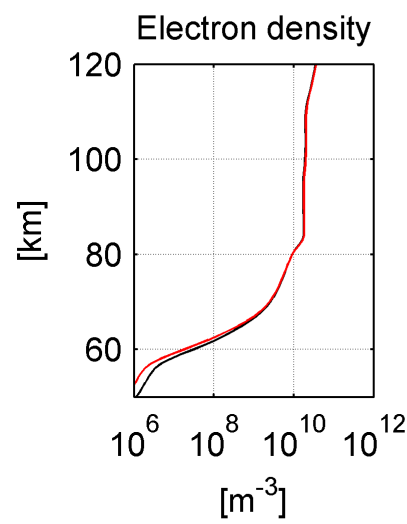

Relative change [\%]

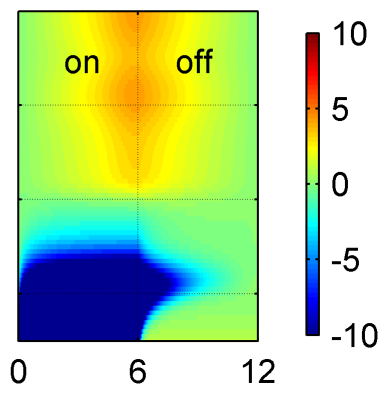

[s]

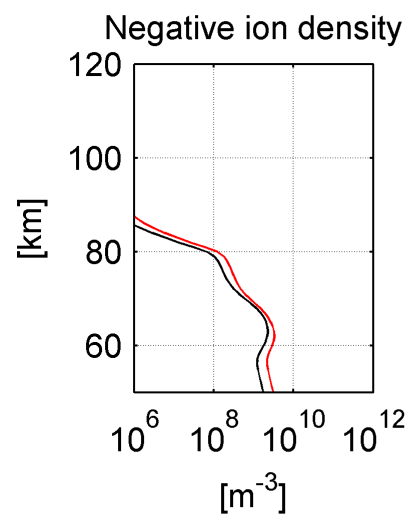

Relative change [\%]

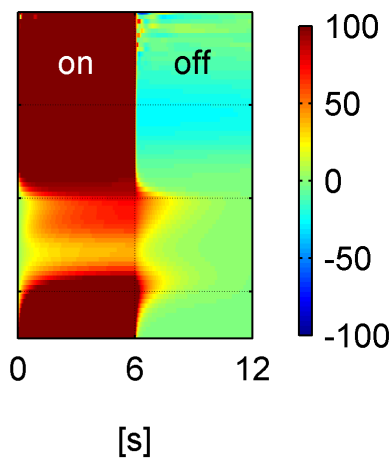

Fig. 3. Ion chemistry response to the 6-s on/off heating ( $T_{e}$ is $5 T_{n}$ during the on- and $T_{n}$ during the off-period). Panels on the left side: electron and negative ion density difference between the ends of the on- and off-periods (red and black lines respectively). Panels on the right: relative change of the electron and negative ion density as a function of time.

the off-period value, which confirms the first findings of the heater induced PMWE modulation (Kavanagh et al., 2006).

\section{Data diagnostic methods}

For quantifying the heating effect found, an exponential ACF theory is applied to the data by using the MCMC inversion. The SIC model is used for selecting required prior probability distributions for ionospheric parameters, and moreover, to parameterise the ion chemistry response to the heating (Figs. 2 and 3). The behaviour of the theory, as a function of heating effect magnitude, is summarised in Fig. 4 for the six cases studied. As a comparison, the results of the theoretical heating model are also shown in Fig. 4.

\subsection{Exponential ACF of a collisional plasma}

According to Fukuyama and Kofman (1980), the ion-line part of the IS spectrum can be approximated as Lorentzian 

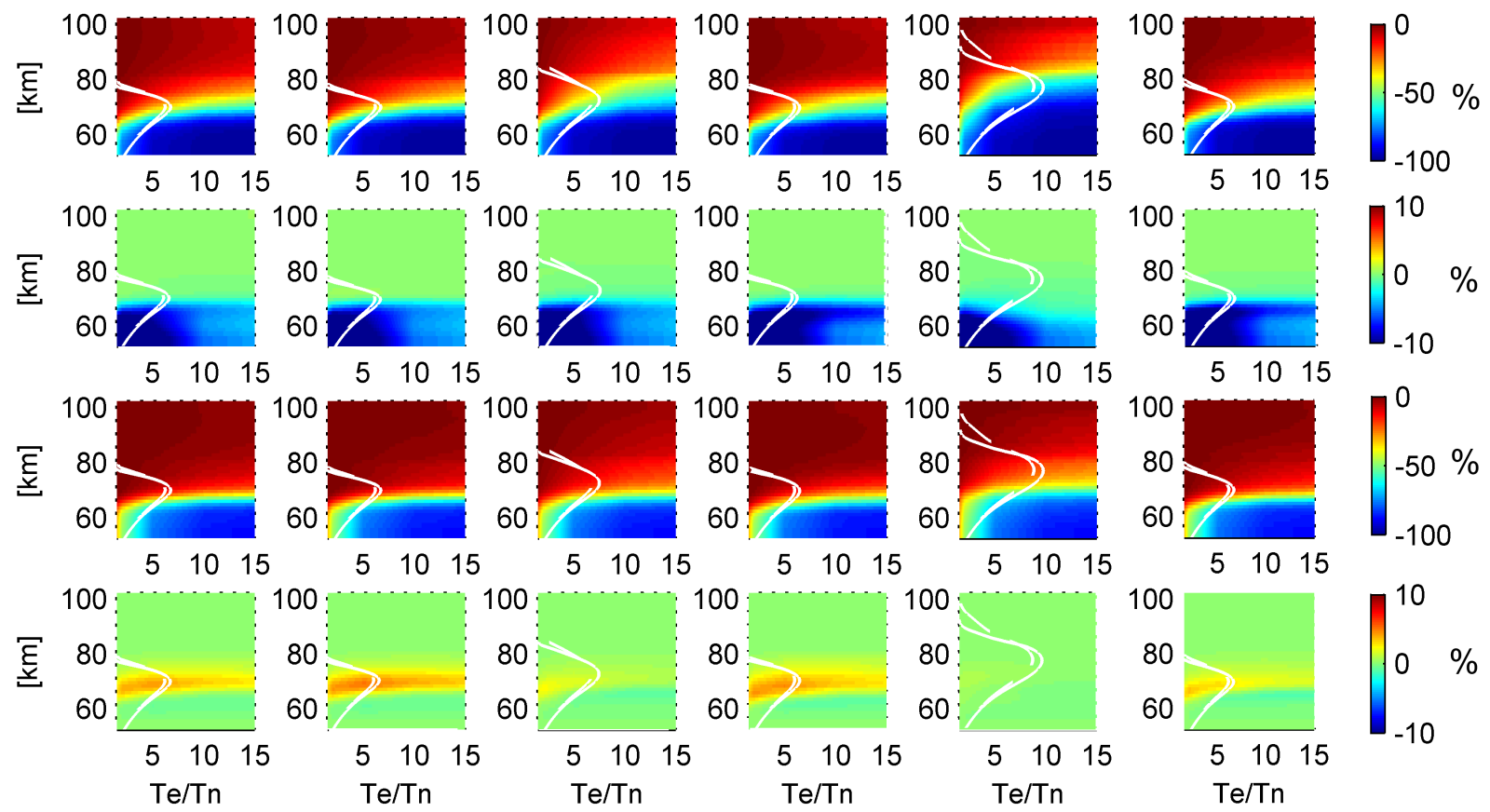

Fig. 4. Modelled IS power and spectral width difference between the 6-s heater-on and heater-off periods as a function of the on-period $T_{e} / T_{n}$-ratio. The modelling is done for the six cases studied (columns 1-6). First row of panels: relative change of the IS power. Second row: change of the IS power related to ion chemistry change. Third row: relative change of the spectral width. Last row: chemistry part of the spectral width change.

shaped in the case of a collisional plasma. If the total backscattered power is $P$, and the spectral line half-width at half-maximum (HWHM) is $\Delta f$, the Lorentzian spectrum $s$ takes the form

$s(f)=\frac{P}{\pi}\left(\frac{\Delta f}{(f-\delta f)^{2}+\Delta f^{2}}\right)$,

where $f$ is the frequency difference with respect to the radar carrier wave and

$\delta f=v / \lambda_{r}$

is the Doppler shift due to the plasma drift velocity $v$ towards the radar operating at wavelength $\lambda_{r}$. Applying the Inverse Fourier Transform to Eq. (1) yields a complex exponential autocorrelation function

$a(\tau)=P \exp \left(2 \pi \tau\left(i v / \lambda_{r}-\Delta f\right)\right)$.

The backscattered power $P$ (Fukuyama and Kofman, 1980) and the spectral width $\Delta f$ (Fukuyama et al., 1987) can be expressed as

$$
P=\frac{2 N_{e}(1+2 \lambda)}{\left(2(1+\lambda)+\left(4 \pi \lambda_{D} / \lambda_{r}\right)^{2}\right)\left(1+\left(4 \pi \lambda_{D} / \lambda_{r}\right)^{2}\right)}
$$

and

$\Delta f=\frac{8 \pi k_{B} T_{n}}{\lambda_{r}^{2} m_{i} v_{i n}} \cdot \frac{\left[2(1+\lambda)+\left(4 \pi \lambda_{D} / \lambda_{r}\right)^{2}\right]}{\left[1+\left(4 \pi \lambda_{D} / \lambda_{r}\right)^{2}\right]}$ as a function of the Debye length

$\lambda_{D}=\sqrt{\frac{\varepsilon_{0} k_{B} T_{e}}{N_{e} q_{e}^{2}}}$.

The parameters and their symbols used in Eqs. (4), (5) and (6) are electron density $N_{e}$, negative ion to electron density ratio $\lambda$, Boltzmann's constant $k_{B}$, neutral temperature $T_{n}$, mean ion mass $m_{i}$, electron to ion collision frequency $v_{i n}$, vacuum permittivity $\varepsilon_{0}$, electron temperature $T_{e}$ and unit charge $q_{e}$.

In this study, the IS power in Eq. (4) is normalised to approach $N_{e}$ in the limit of $\lambda \rightarrow 0$ and $\lambda_{D} / \lambda_{r} \rightarrow 0$. This makes it possible to apply the theory directly to the calibrated ACF measurements described in Sect. 2.

The IS spectrum depends on the prevailing electron temperature via the Debye length (see Eq. 6). In addition, electron temperature dependent chemistry determines the parameters $N_{e}$ and $\lambda$. Parameterising the chemistry response to the heating is considered in the following section.

\subsection{Sodankylä Ion Chemistry model (SIC)}

The Sodankylä Ion Chemistry (SIC) model is a detailed 1-dimensional time-dependent chemistry model, which includes 36 positive ions, 27 negative ions and $14 \mathrm{mi}-$ nor neutrals coupled together via more than 300 reactions 
(Verronen, 2006). The model uses altitude profiles of neutral temperature and major atmospheric species from the empirical MSISE-90 model (Hedin, 1991) and from Shimazaki (1984).

In the present work an experimental version (SIC 7.2), developed as part of the work presented by Enell et al. (2008), was applied for modelling the background altitude profiles of $N_{\mathrm{e}}, \lambda$ and $m_{\mathrm{i}}$. Only solar radiation and electron precipitation were considered as ionisation sources. The calculation of photoionisation and electron ionisation rates follows Rees (1989). Solar spectra are taken from the SOLAR 2000 model (Tobiska et al., 2000). For the electron precipitation an angularly isotropic Maxwellian electron energy distribution is assumed. The precipitation parameters were chosen for the IS power (Eq. 4) profile to match the measured IS power (see Fig. 2).

In addition to the background condition modelling, SIC 7.2 allows modelling the ion-chemical response to heating by setting the electron temperature independently from the neutral temperature in 13 electron attachment, electron detachment and ion-electron recombination reactions. In the cases studied a parameterisation using $T_{\mathrm{e}} / T_{\mathrm{n}}$ ratios of 1.5 , 2, 5, 10, 30 and 100 was adopted, and the effects were interpolated between these values. Figure 3 shows the modelled changes for negative ion and electron densities when the electron temperature is increased in $6 \mathrm{~s}$ periods $\left(T_{\mathrm{e}}=5 T_{\mathrm{n}}\right.$ at all altitudes) and the reactions allowed to recover for the same period ( $w i t h T_{\mathrm{e}}=T_{\mathrm{n}}$ ). A clear electron density decrease is visible during heating below $70 \mathrm{~km}$ due to the increased rate of electron attachment producing negative ions. On the other hand, the electron density increases above $80 \mathrm{~km}$ due to a decrease in the electron recombination rate.

Figure 4 shows the modelled IS spectral response to the heating for the 6 cases studied. It turns out that the heatinginduced increase in negative ion production can have a significant effect on the IS power and spectral width at the altitudes of expected maximum heating. In contrast, the electron density increase above $80 \mathrm{~km}$, associated with the change of the recombination rate, has no detectable effect on the modelled ACF in the cases studied.

In the analysis, the electron temperature is deduced from the detected ACF profiles based on the Debye length and the change in chemistry. The actual fitting procedure is considered in the following section.

\subsection{The Markov Chain Monte Carlo (MCMC) method}

The Metropolis-Hastings Markov Chain Monte Carlo (MCMC) inversion method (Hastings, 1970) is used for fitting the theory to the measured ACFs shown in Fig. 1. The method uses a random sampling of a given prior distribution providing as a result a posteriori distribution for each of the fitted parameters. Gaussian prior distribution around the SIC-modelled mean value was used for the parameters $N_{e}$ (variance $\sigma=0.2), \lambda(\sigma=0.2)$ and $m_{i}(\sigma=0.1)$. The neutral atmosphere MSIS-90 model was used for obtaining flat prior distributions of $T_{n}$ and $v_{i n}$ (from 0.8 to 1.2 times the modelled value) and heated $T_{e}$ (from 0.9 to 50 times the modelled $\left.T_{n}\right)$.

In each round of the MCMC iteration, the time evolution of the ACF was calculated over the 6-s on/off cycle, based on the SIC-modelled chemical response. These time-averaged ACFs were compared to the data (o-mode, $\mathrm{x}$-mode and off separately) until the routine converged around a set of most probable posteriori values. The posteriori distributions for the electron temperature estimates are considered as final results in Sect. 4.

\subsection{Heating model}

Modelling of the electron temperature increase due to the HF heating is based on finding thermal equilibrium between the ohmic heating of the electron gas and the electron energy loss associated with excitation processes. The principle follows Belova et al. (1995) and a detailed description of the present model can be found in Kero et al. (2007). The modelled electron temperature enhancements are shown in Fig. 4 and 5 for the six cases studied. The maximum effect appears in altitudes of $70-75 \mathrm{~km}$, where the modelled $T_{e} / T_{n}$-ratio varies between 6 and 8 . Typically, the modelled x-mode effect is slightly stronger and limited in lower altitudes compared to the o-mode effect. The difference between the modes, however, is small in the cases corresponding to high D-region electron densities (cases 1, 2, 4 and 6).

It is worth mentioning that the electron density change due to heating (see Fig. 3) modifies slightly the opacity of the plasma for the radio wave and could therefore cause a secondary effect in the modelled electron temperature profile. However, this effect turns out to be negligibly small less than 3 percent of the electron temperature at the heating maximum. Hence, it is justified to consider a constant electron temperature profile over the heated period. In the off periods, the electron temperature is assumed to be equal to the neutral temperature.

\section{Results}

Figure 5 shows MCMC posteriori distributions of the electron temperature for each of the six cases studied. It turns out that the theoretical approach described in the previous section was able to produce a reasonable set of stable temperature estimates only in three cases out of six, corresponding to the high SNR conditions (see Fig. 2). Moreover, the successful quantifications were limited to the 68 to $88 \mathrm{~km}$ altitude range.

The modelled IS power and spectral width results in Fig. 4 show that the ACF is insensitive to the electron temperature enhancements at high altitudes. This yields a growth of temperature estimate variances at the upper $\mathrm{D}$ region seen in 

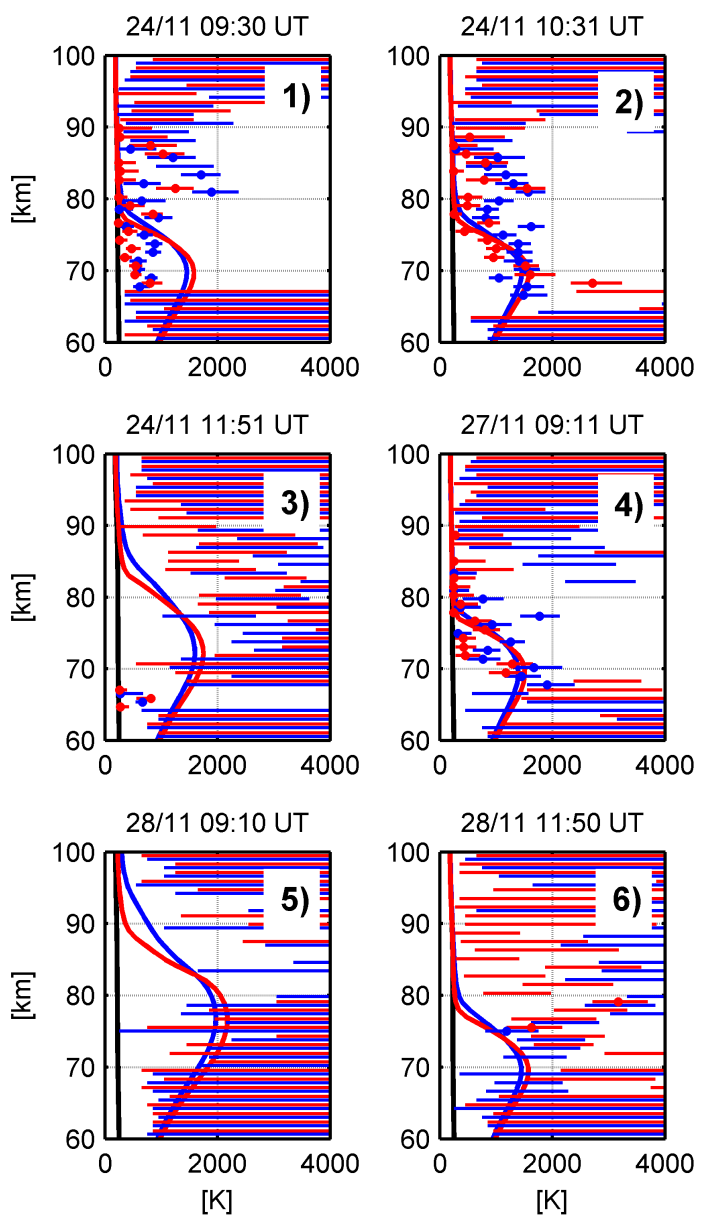

Fig. 5. Electron temperature estimates compared to heating model results (blue: o-mode, red: x-mode, black: heater off). Horizontal lines represent 90 percent significance widths of the MCMC posteriori distributions. Most probable values are marked with dots when the distribution width is less than $1000 \mathrm{~K}$. Continuous lines show modelled temperature profiles.

Fig. 5 results. High variance is also present at the lowest measured altitudes due to the weak backscattered power. Therefore, the successful temperature estimates take place in between these regions under suitable conditions (cases 1, 2 and 4).

For comparison, a reference data analysis was done without the chemistry parametrisation described in Sect. 3.2. It turns out that disregarding the negative ion production effect on the theoretical ACF causes an increase in the electron temperature estimates at lower D-region altitudes. At the heating maximum, this bias is of order of a few hundred Kelvins increasing towards the lowest altitudes of the experiment up to $1000 \mathrm{~K}$.

There is a fairly good agreement between the theoretical heating model and the data in cases 2 and 4 at the altitudes of the maximum effect. On the other hand, an unexpectedly weak effect is found in case 1 , which could be associated with the strong PMWE. In fact, the overall difference between the heated and the unheated ACF is not as clear in case 1 as it is, for example, in case 2, which represents approximately the same ionospheric conditions one hour later, but without a strong PMWE (Fig. 1).

In addition, there is a second maximum of the heating effect between $80-90 \mathrm{~km}$ in the cases 1,2 and possibly 4 . This feature is not predicted by the model.

\section{Conclusions}

The new VHF-radar experiment sippi was optimised to probe the artificially heated D-region ionosphere for the EISCAT campaign in November 2006. The recorded data shows systematic differences in the received IS signal between heated and unheated periods. The IS data analysis method presented interprets the differences as caused by electron temperature dependent Debye-length and ion chemistry changes. The latter turns out to be important only at lower D-region altitudes.

The method was able to estimate the increased electron temperature in three data examples out of six. These are the first successful IS measurements in the altitudes of the expected maximum effect. A good agreement with the theoretical heating model was found in two cases, while in one of the cases the model overestimates the effect.

Acknowledgements. Thule institute/University of Oulu supported the main author's work by EnviroNet grant. Juha Vierinen is funded by the Academy of Finland (application number 213476, Finnish Programme for Centres of Excellence in Research 2006-2011). Carl-Fredrik Enell is funded by the Academy of Finland through project 109054, Solar Energetic Radiation and Chemical Aeronomy of the Mesosphere. Ilkka Virtanen is funded by Finnish Graduate School in Astronomy and Space Physics. The EISCAT measurements were made with special programme time granted for Finland. EISCAT is an international assosiation supported by China (CRIRP), Finland (SA), Germany (DFG), Japan (STEL and NIPR), Norway (NFR), Sweden (VR) and United Kingdom (STFC).

Topical Editor K. Kauristie thanks M. Jarvis and M. Kosch for their help in evaluating this paper.

\section{References}

Bailey, V. A. and Martyn, D. F.: Interaction of radio waves, Nature, 133, 218, 1934.

Barr, R., Rietveld, M. T., Kopka, H., and Stubbe, P.: The effect of a heated patch of auroral ionosphere on VLF radio wave propagation, Nature, 309, 534-536, 1984.

Belova, E. G., Pashin, A. B., and Lyatsky, W. B.: Passage of a powerful HF radio wave through the lower ionosphere as a function of initial electron density profiles, J. Atmos. Terr. Phys., 57, 265272, 1995.

Chilson, P. B., Belova, E., Rietveld, M. T., Kirkwood, S., and Hoppe, U.-P.: First artificially induced modulation of PMSE us- 
ing the EISCAT heating facility, Geophys. Res. Lett., 27, 38013804, 2000.

Enell, C.-F., Verronen, P. T., Beharrell, M. J., Kero, A., Seppälä, A., Ulich, T., Turunen, E., and Honary, F. H.: Case study of the mesospheric and lower thermospheric effects of solar X-ray flares: Coupled ion-neutral modelling and comparison with EISCAT and riometer measurements, Ann. Geophys., in press, 2008.

Fukuyama, K. and Kofman, W.: Incoherent scattering of an electromagnetic wave in the mesosphere: A theoretical consideration, J. Geomag. Geoelectr., 32, 67-81, 1980.

Fukuyama, K. Y., Maekawa, S., and Fukao, S. Kato, S.: Ionospheric D-region temperatures, and electron and neutral densities observed by the incoherent scatter technique at Arecibo, Ann. Geophys., 5A, 289-296, 1987.

Hastings, W. K.: Monte Carlo Sampling Methods Using Markov Chains and Their Applications, Biometrika, 57, 97-109, doi:10. 2307/2334940, 1970.

Hedin, A. E.: Extension of the MSIS Thermospheric Model into the Middle and Lower Atmosphere, J. Geophys. Res., 96, 11591172, 1991.

Kavanagh, A. J., Honary, F., Rietveld, M. T., and Senior, A.: First observations of the artificial modulation of polar mesospheric winter echoes, Geophys. Res. Lett., 33, L19 801, doi:10.1029/2006GL027565, 2006.

Kero, A., Bösinger, T., Pollari, P., Turunen, E., and Rietveld, M.: First EISCAT measurement of electron-gas temperature in the artificially heated D-region ionosphere, Ann. Geophys., 18, 12101215, 2000, http://www.ann-geophys.net/18/1210/2000/.

Kero, A., Enell, C.-F., Ulich, T., Turunen, Rietveld, M., and Honary, F. H.: Statistical signature of active D-region HF heating in IRIS riometer data 1994-2004, Ann. Geophys., 25, 407-415, 2007, http://www.ann-geophys.net/25/407/2007/.

Rees, M. H.: Physics and Chemistry of the Upper Atmosphere, Cambridge University Press, Cambridge, UK, 1989.
Rietveld, M. T., Kopka, H., and Stubbe, P.: D-region characteristics deduced from pulsed ionospheric heating under auroral electrojet conditions, J. Atmos. Terr. Phys., 48, 311-326, 1986.

Rietveld, M. T., Kohl, H., Kopka, H., and Stubbe, P.: Introduction to ionospheric heating at Troms $\varnothing$-I. Experimental overview, J. Atmos. Terr. Phys., 55, 577-599, 1992.

Rietveld, M. T., Turunen, E., Matveinen, H., Goncharov, N. P., and Pollari, P.: Artificial periodic irregularities in the auroral ionosphere, Ann. Geophys., 14, 1437-1453, 1996, http://www.ann-geophys.net/14/1437/1996/.

Shimazaki, T.: Minor Constituents in the Middle Atmosphere, no. 6 in Developments in Earth and Planetary Physics, D. Reidel Publishing Company, 1984.

Stubbe, P., Kopka, H., Rietveld, M. T., and Dowden, R. L.: ELF and VLF wave generation by modulated heating of the current carrying lower ionosphere, J. Atmos. Terr. Phys., 44, 1123-1135, 1982.

Tobiska, W., Woods, T., Eparvier, F., Viereck, R., Floyd, L., Bouwer, D., Rottman, G., and White, O.: The SOLAR2000 empirical solar irradiance model and forecast tool, J. Atmos. SolarTerr. Phys., 62, 1233-1250, 2000.

Turunen, E., Matveinen, H., Tolvanen, J., and Ranta, H.: D-Region Ion Chemistry Model, in: STEP Handbook of Ionospheric Models, edited by: Schunk, R. W., pp. 1-25, Scientific Committee on Solar-Terrestrial Physics, 1996.

Verronen, P. T.: Ionosphere-atmosphere interaction during solar proton events, Ph.D. thesis, Finnish Meteorological Institute, Helsinki, Finland, http://ethesis.helsinki.fi/, ISBN: 951-697-6506, 2006.

Virtanen, I. I., Lehtinen, M. S., Nygren, T., Orispaa, M., and Vierinen, J.: Lag profile inversion method for EISCAT data analysis, Ann. Geophys., 26, 571-581, 2008, http://www.ann-geophys.net/26/571/2008/. 